\title{
IOT Based Patient Monitoring System
}

\author{
Hemalatha Rallapalli ${ }^{1}$, Pavani Bethelli ${ }^{2}$
}

\begin{abstract}
Nowadays, hospitals across the country are preparing themselves for predicting provider shortages. Patients are facing long waits between appointments or before appointments, including delays in being moved between different departments and inadequate response to requests or complaints. In this paper, a smart health care system is proposed, that consists of a smart RFID tag, local server, internet, medical server and service provider. Smart RFID tag provides physiological condition (medical record) of the patient and that medical record is diagnosed by the doctor through WLAN. The objective of this paper is monitoring of patient regularly.
\end{abstract}

Keywords-Sensor, RFID tag, WLAN.

\section{INTRODUCTION}

The healthcare sector in the world is facing a number of challenges like increasing population, aging infrastructure, immense masses of rural population with limited access to high-quality medical care and a disproportionate number of medical staff when compared to the huge population. It is the need of the day to design new techniques to improve the healthcare systems in the world. Implementation of the health care system is an ongoing process which includes several stages. One of the ongoing processes includes smart health care system which is achieved by the internet to facilitate the common man with limited investment.

Health is one of the global challenges of humanity. Healthy individual leads the life happily and securely. Healthy individual reduces the burden on the hospitals, clinics and medical professionals etc. It increases individual income and hence to increase in global domestic product and in tax revenues. The modernized healthcare system is a prerequisite to keeping individuals healthy. The current healthcare system facing two problems.

- The healthcare professionals and staff must be on a site of the patient all the time.

- The patient remains admitted in a hospital, wired to bedside biomedical instruments, for a period of time.

Modernized healthcare system or smart healthcare system has been implemented to solve these two problems. Disease diagnosis, prevention, and patient monitoring are easy by using the smart healthcare system.

The proposed system consists of three phases:

- Data acquisition phase for monitoring and transferring the patient data.

- Data transfer for transmitting the signals between the sensors and Hospital network.
- Data access for accessing and monitoring the patient data.

The healthcare domain presents opportunities for a significant number of applications of wireless sensor technology. The following sections focus on Chronic Disease Monitoring and Personal Wellness Monitoring.

\section{Proposed HealthCare System}

Functions of a smart system include sensing, actuation and controlling. It makes the decisions based on available data in a predictive or adaptive manner by performing smart actions. In the healthcare sector, smart systems technology leads to Disease diagnosis, prevention, and patient monitoring is easy. It provides quality of service and reduces the cost of the public healthcare system. Proposed healthcare system block diagram as shown in fig.1 [4].

The smart RFID tag attached to the patient. Smart RFID tag consists of several sensors [5]. The system uses biosensors like Temperature, blood pressure, weight sensors etc. The flow chart of smart RFID tag as shown in figure 2. Whenever the patient comes to the hospital premises, sensors sense the physiological signals and these signals are converted to electrical signals [6]. Then analog electrical signal is converted to digital signal (digital data) which is stored in RFID.

The stored digital data is transmitted through Zigbee protocol [7] to the local server Zigbee is suitable protocol for this system because it is IEEE 802.15.4 standard protocol. It consists of maximum number of cell nodes. IEEE 802.15.4 standard protocol (Zigbee) is designed for wireless networking among sensors. It is more preferred for devices which are smaller in size and consume less energy.

From local server the data is transferred to the medical server through WLAN which is based on IEEE 802.11 standard [8]. Medical server consists of large database. When the data is transferred to the medical server, it checks whether the patient has any previous medical record then the server adds the new data to that record and transfers to the doctor. If patient does not have any past medical record then the server creates new ID and stores the data in its database. This data is transferred to the doctor.

The doctor receives patient's profile from the medical server and identifies the problem. If patient condition is normal then doctor gives prescription. This prescription is available in local server. If the patient condition is abnormal, doctor advice the patient and monitor accordingly. Fig. 3 shows the flow chart of diagnosis

\footnotetext{
${ }^{1}$ University college of engineering, Osmania university

${ }^{2}$ Teegala Krishna Reddy College Of Engineering
} 


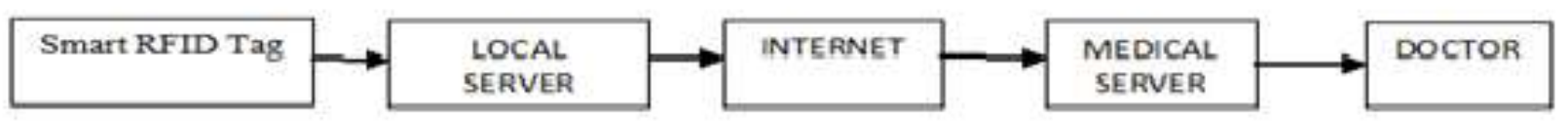

Fig1. Block diagram of smart healthcare system

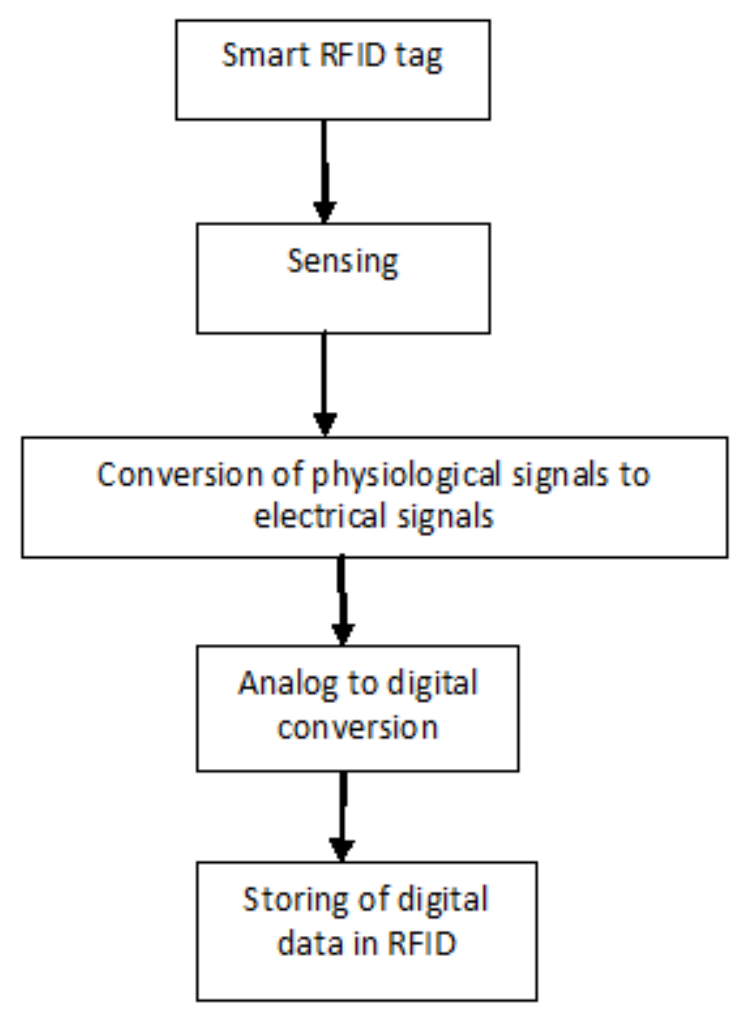

Fig. 2. Flowchart of smart RFID Tag

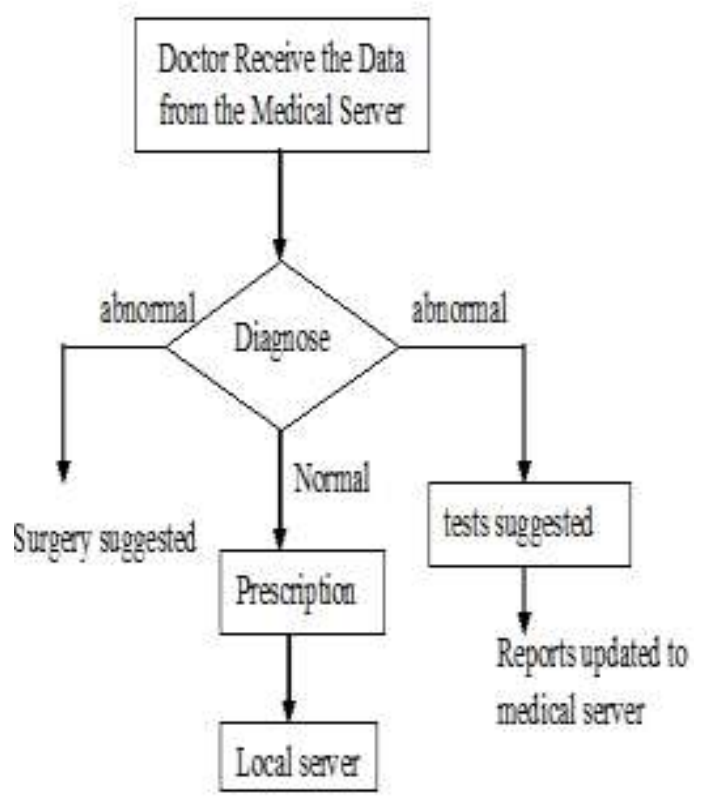

Fig.3 Diagnosis
Suppose patient condition is abnormal and doctor suggested surgery. Here patient monitoring is done in two levels

- Pre surgery monitoring

- Post surgery monitoring

\subsection{Pre Surgery Monitoring}

Advancement in technology, improved health care system. In this system, using smart RFID tag patient is monitored continuously. Smart RFID tag consists of several sensors like blood pressure, ECG, blood glucose, temperature sensor etc.

\section{- ECG Sensor}

One of the sensors in Smart RFID tag is the ECG Sensor. The ECG sensor is attached to the patient using disposable electrodes on the chest. It senses the physiological signals which are produced during muscle contractions and can be used to make standard 3-lead ECG tracings or surface EMG recordings[9] [10]. The signal obtained from the body is converted into an electrical signal which is passed through the filter, amplifier and the analog-to-digital converter (ADC). The serial-to-ZigBee module transmits the digital output of the ADC to the local server.

- Blood Glucose Sensor

Blood glucose sensor is one of the major biosensors. Glucose sensor consists of a tiny electrode which is inserted under the skin.It senses the physiological signal and which is converted into a digital signal(digital data) and that data is transmitted to medical server.CGM (continuously glucose monitoring)[11] is one of the blood glucose sensors and it is used to measure glucose levels in real-time throughout day and night.

Flow chart of pre surgery monitoring as shown in fig.4

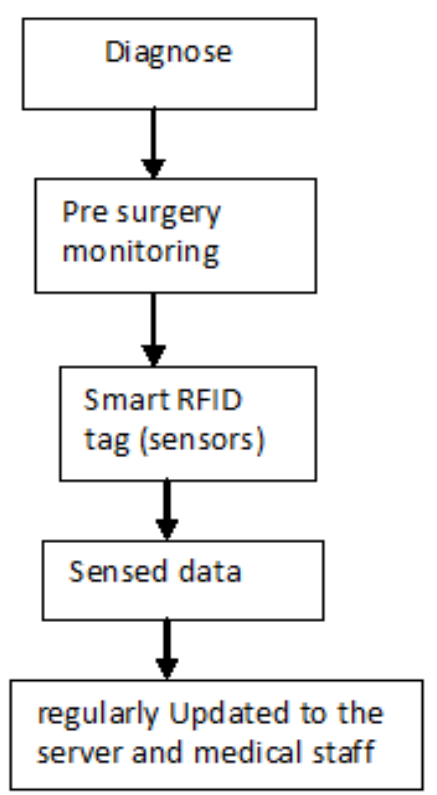

Fig 4. Pre surgery monitoring 


\subsection{Post Surgery Monitoring}

Monitoring of patient after surgery is crucial part of this system. Recovery of the patient after post surgery will vary depending on the type of procedure done and anesthesia used. Total body conditioning after the surgery will be monitored by using this proposed system.

For example after completion of surgery the patient is moved to the general ward. Let the ward consist of six numbers of patients. Figure 5 shows post surgery monitoring room and that room has smartly connected system that means everywhere sensors are connected. Each Patient wears the smart RFID tag and it consist of several sensors. In addition to the normal sensors (temperature, BP etc) other special sensors also used to monitor the patient. Those sensors are

- Dehydration Sensor

Dehydration sensor is one of the wearable sensors and it sense when the patient is dehydrated. Wrist band type of dehydration sensor consist of tiny micro-needles and these is sticks into the skin to measure fluid levels [13].

\section{- Pulse Rate Sensor}

The pulse rate sensor or heart beat sensor is based on the principle of photo phlethysmography [12]. It is used to measure the number of pulses produced by the heart. According to this principle, when the light falls on any organ, it changes the volume of the blood through that organ. Changes in light intensity are proportional to change in volume of the blood. The change in a volume of the blood decided by the rate of heart pulses. Whenever the light is absorbed by blood, the signal pulses are equivalent to the heart beat pulses.

- Flow Sensor (IR Sensor)

A flow sensor is a device for sensing the rate of fluid flow. IR sensor is used to detect the droplet coming from the saline. It alerts the medical staff when it crosses the threshold level.
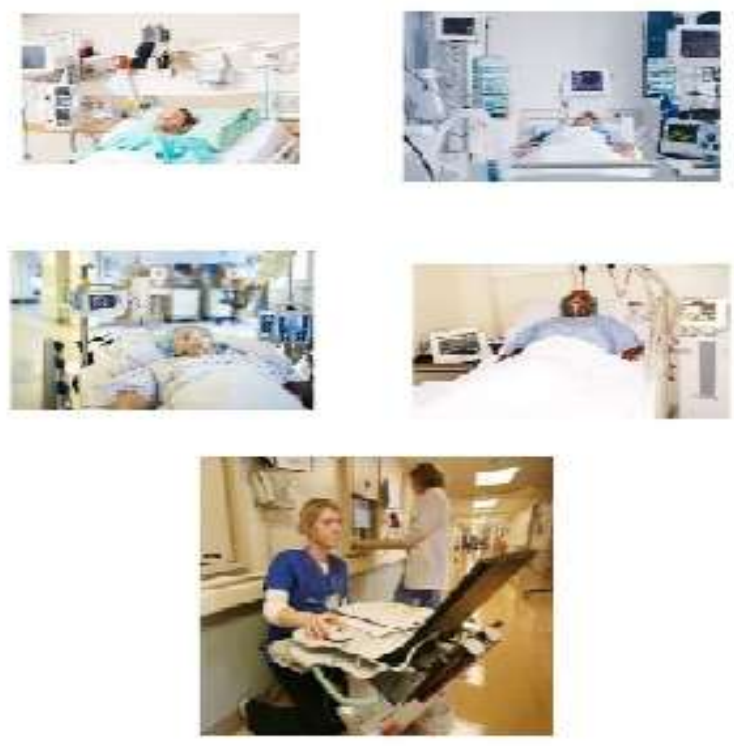

Fig 5. Post Surgery Monitoring Room
Sensors sense the signals and these signals are converted to electrical signals. Then analog electrical signals are converted to digital signals (digital data), which is stored in RFID. Medical staff receives monitored data continuously without any physical observation. Figure 6 shows the flow chart of post surgery monitoring.

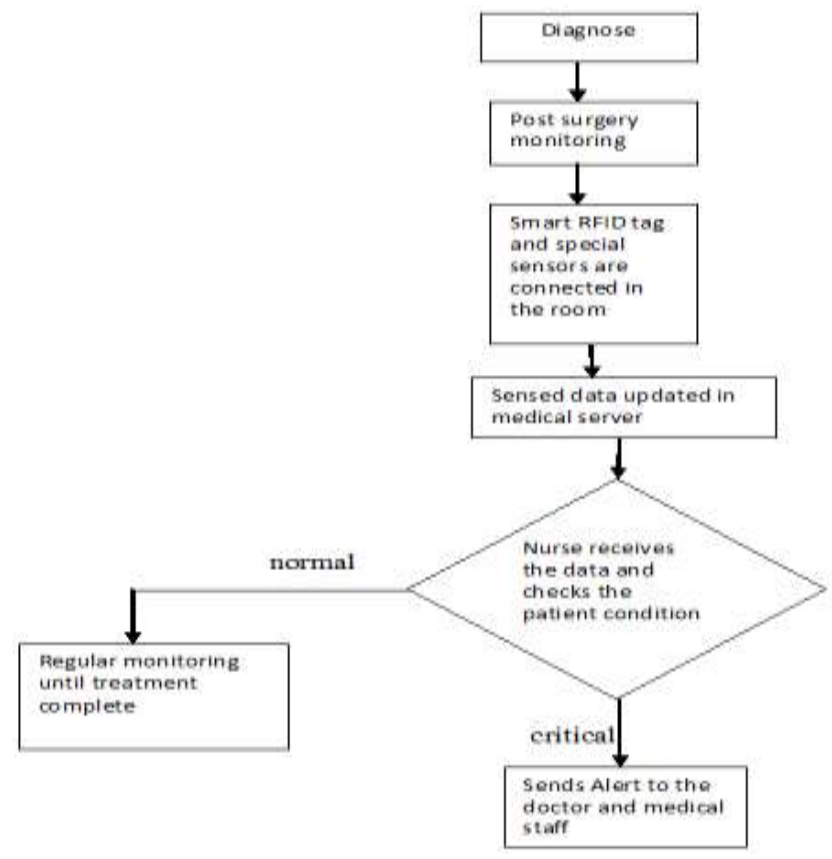

Fig 6. post surgery monitoring

\section{CONCLUSION}

Here, we proposed a mobile physiological monitoring system, which is able to continuously monitor the patient's temperature, blood pressure, heart beat, blood glucose level and other critical parameters in the hospital. This paper demonstrates the use of smart healthcare system. This new technology has potential to offer a wide range of benefits to patients, supervised rehabilitation, reducing of long waits, early detection of abnormal conditions, continuous patient monitoring, and potential knowledge discovery through data mining of all gathered information.

\section{REFERENCES}

[1] C.D.Mathers and D. Loncar, " projections of global mortality and burden of disease from 2002 to 2030," PLOS Med, vol.3,no.11,pp. 2011-2029,nov.2006.

[2] X. F. Teng and Y. T. Zhang, "M-health: Trends in wearable medical devices," Chinese J. Med. Instrum., vol. 30, no. 5, pp. 330-340, Sep. 2006.

[3] M. Scholtz, "Addressing the global demands for improved healthcare," in Proc. Telemedicine 21 st Century, Opportunities Citizens, Society, Industry, 1999, pp. 11-18.

[4] Prosanta Gope and Tzonelih Hwang, "BSN-Care: A Secure IoT-Based Modern Healthcare System Using Body Sensor Network,", IEEE Sensors journal vol.16,no.5,pp. 1368-1376, march 1, 2016.

[5] Corrado fellini, Massimo Merenda and Francesco G. Della Corte, "Battery-less smart RFID tag with sensor capabilities," RFIDTechnologies and Applications (RFID-TA), 2012 IEEE International Conference ,pp. 160-164. 
[6] Shubash Chandra mukhopadyaya,"Wearable Sensors for Human Activity Monitoring," IEEEsensors journal vol.15,no.3,pp.1321-1327, march,2015.

[7] Zigbee Alliance.(2009,jun.15).[online].Available: www.zigbee.org.

[8] IEEE standards association [online].available: standards.ieee.org.

[9] Elisa Spanò; Stefano Di Pascoli; Giuseppe Iannaccone," Low-Power Wearable ECG Monitoring System for Multiple-Patient Remote Monitoring,”, IEEE Sensors Journal Year: 2016, Volume: 16, Issue: 13Pages: $5452-5462$.

[10] Wan-young chung, young-dong lee and sang-joong jung," A wireless sensor network compatible wearable U-healthcare monitoring system using integrated ECG, accelerometer and SPO2", IEEE EMBS conference-2008, pp. 1529-1532.

[11] IEEE Draft for Health Informatics - Personal Health Device Communication - Part 10425: Device Specialization Continuous Glucose Monitor (CGM) - Corrigendum 1: year 2016. Pp.1-33.

[12] T. Tamura, Y. Maeda, M. Sekine, and M. Yoshida, "Wearable photoplethysmographic sensors-Past and present," Electronics, vol. 3, no. 2,pp.282-302, 2014. 